\title{
LOW-VOLTAGE THIN-FILM PIEZOELECTRIC SENSE-ACTUATE FAN PAIR FOR CHIP-SCALE GAS-SENSOR
}

\author{
V. Gund ${ }^{* 1}$, L. Sanchez ${ }^{2}$, R.G. Polcawich ${ }^{2}$, and A. Lal ${ }^{1}$ \\ ${ }^{1}$ SonicMEMS Laboratory, Cornell University, USA \\ ${ }^{2}$ Army Research Laboratory, USA
}

\begin{abstract}
Chip-scale gas sensing devices based on ion-mobility measurements of analytes offer the potential for fast and reliable detection of toxic compounds and chemical warfare agents, which maybe odorless or colorless. The ability to produce required ionflow rates using low-power architectures is critical in order to maximize sampled air volume for fast detection. In this paper, we present a novel thin-film piezoelectric sense and actuate pair of fans as a component for controlled ion flow in ion-mobility-based gas detectors. The drive-fan achieves low-power operation (13.5 $\mathrm{mW} / \mathrm{sccm})$, low voltage $(10.8 \mathrm{~V} / \mathrm{sccm})$ with sufficiently small form-factor $\left(0.1 \mathrm{~mm}^{3} / \mathrm{sccm}\right)$ along with feedback flow signal from the sense-fan to measure the flow and detect the onset of turbulent flow. This device has the potential for monolithic integration with CMOS devices for chip-scale label-free gas detection.
\end{abstract}

\section{INTRODUCTION}

Micro-scale gas pumping devices are important components of portable gas-sensors and gas chromatographs for emission content monitoring in industry, automation and food packing as well as environmental sensors for maintaining air quality [1]. Gas pumps also find applications in microfluidic devices for laminar flow control and manipulation of gases in microfluidic fuel cells with precise mass transport requirements to the electrodes, to reduce electrode size and improve power density [2]. Furthermore, convective air-flow generation for cooling and thermal management of electronic devices is a growing area of interest in order to optimize overall power requirements without affecting device performance [3].

In ion-mobility gas sensors, high ion-flow rates are often required without any turbulence. To accomplish this, inertial fluid forces must be weaker than viscous damping forces in order to produce laminar flow in micro-channels. Centrifugal pumps using scratch-drive rotary fans have been used for air-flow generation via conversion of rotational kinetic energy to hydrodynamic energy of air [4]. These fans have resonant frequencies in a few-kHz range and require $30 \mathrm{~V}_{\mathrm{p}-\mathrm{p}}$ operation but are prone to frictional wearing inherent in their operational mechanism. In order to achieve high flow rates and large pressure differentials on chip for gaschromatographs and ion-mobility based sensors, micro-valve based multi-stage gas pumps in mm-scale packages have been extensively explored [5]. To minimize device footprint and complexity for integration in miniaturized micro-packages, torsional axial fans based on resonant electrostatic actuation have been used to produce flow rates of up to $25 \mu \mathrm{L} / \mathrm{min}$ using arrays of fans [6]. However, operation voltages required for electrostatically actuated device vary from 100-150 V.

Axial piezo-electric fans provide a low-power and lowvoltage solution for chip-scale ion flow generation. Bulk lead zirconate titanate (PZT) plates with mylar or steel plates attached to them have been previously used for cooling of electronic devices by convective removal of heat in confined spaces [3]. Such a fan has low-power consumption and low-noise operation. However, the large size is not suitable for ultra-portable packaged sensors, used in burgeoning handheld smart-phone and tablet markets. This work presents the design, analysis and characterization of a novel thin-film sense-actuate piezo-fan pair for chip-scale gas-sensors.

A schematic of the fan-pair with the gas-sensing system is shown in Figure 1. We have previously demonstrated a laser micro-machined low-voltage ion-mobility spectrometer (IMS) array [7] which uses lateral electric fields for segregation of analytes on individual electrode islands, and high-aspect ratios for ion-flow confinement in the device channel. The sense-actuate fan pair in this work can be integrated at the input of the IMS channel, as shown, along with radioactive electron-emitting ${ }^{63} \mathrm{Ni}$ for ionizing analytes following initial pre-concentration of analytes, if any. At resonance, the actuation fan produces ion flow via acoustic streaming. For large driving voltage on the actuator, the sense-fan becomes unstable due to the larger random-motion from the flow indicating a threshold, and starts to flutter providing a feedback signal for limiting or eliminating the turbulent flow.

Individually addressed electrode islands and layers separated by insulator

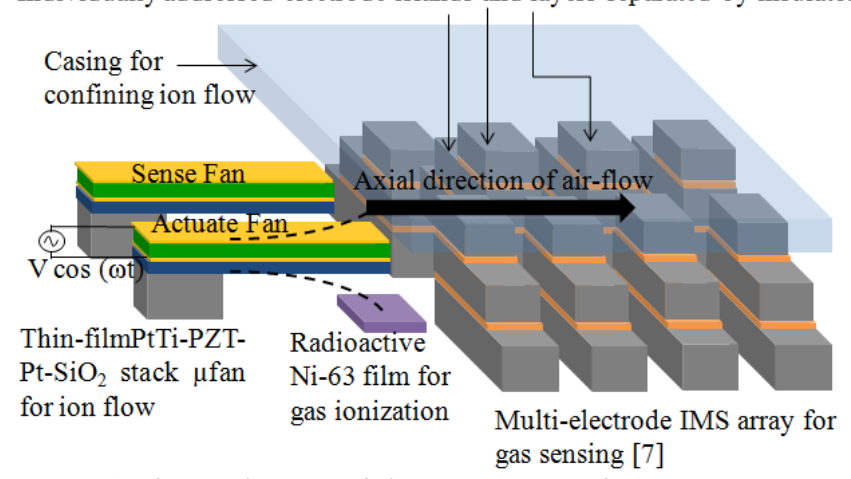

Figure 1:Chip scale ion-mobility gas sensor schematic - IMS array gas sensor, radioactive ${ }^{63} \mathrm{Ni}$ film for gas ionization integrated with piezoelectric sense-actuate microfans for producing ion-flow

\section{PIEZO-FAN MODEL}

Flow generation from oscillating beams has been studied by several authors, particularly for larger beams, but not fully understood due to the complicated non-linear flow dynamics. 2-D flow near the tips of flexible plates with large oscillation amplitudes have been investigated [8] and Toda et al. derived the theory for air-flow generation from a vibrating piezoelectric multimorph under resonant operation [9].

For a piezoelectric film with an applied electric field $E$ the strain vector $s$ is given by -

$$
s=D E
$$

Here, $s$ is a $6 \times 1$ column vector of three axial and shear strains each, $E$ is a $3 \times 1$ column vector of electric-field and $D$ is the $6 \times 3$ coupling coefficient matrix. For our device with metal electrodes patterned on the piezoelectric layer and an elastic layer of $\mathrm{SiO}_{2}$, the $\mathrm{d}_{31}$ component is important for operation - vertical E-field in the PZT produces a longitudinal strain causing the multimorph to bend. The modeling equation of motion for our 4-layer piezoelectric multimorph actuate fan is-

$$
M \ddot{y}+k y+f_{\text {air }}=F \cos (\omega t)
$$


Here, $M$ is the effective mass of the oscillating fan concentrated at the center of the inertial force an effective length $L$ away from the hinge, $k$ is the spring constant of this beam, $f_{\text {air }}$ is the force for driving the air-motion, $F$ is the driving force for the fan by a sinusoidal AC signal for a flat-plate model as described in [9]. The force $f_{\text {air }}$ is usually ignored in resonator analysis as a loss term. However, it is important in our analysis to calculate the streaming flow produced by the fan.

The width of air-region above and below the vibrating fanedge that is streamed radially at resonance $D \approx 2 y_{0}$ where $y_{0}$ is the fan-edge amplitude. A radial torque $T$ is required to accelerate this volume of air. At resonant operation, the air mass directed radially outwards in time $d t$ near the fan edge is $2 \rho_{\text {air }} W y_{0} v / d t$. Here, $\rho_{\text {air }}$ is the density of air $\sim 1.225 \mathrm{~kg} / \mathrm{m}^{3}$, W is the fan width and $v / /$ is the air flow velocity. The time derivative of this mass multiplied by the lever arm i.e. fan length and maximum fan edge velocity is the required torque $T$, and the corresponding $\mathrm{f}_{\text {air }}=T / L^{\prime}$ at the center of the inertial force [9] -

$$
f_{\text {air }}=1.573 \rho_{\text {air }} W D v_{\perp} v_{\amalg}
$$

The numerical factor is a normalization for the distance of the inertial force center from the clamping point. The input mechanical power to the air mass is then -

$$
P_{M}=T \frac{d \theta}{d t}=\rho_{\text {air }} W D v_{\amalg} v_{\perp}^{2}
$$

For ideal coupling, all of this mechanical power is transferred to the air mass and used to produce streaming motion. However, some of this energy is dissipated as heat due to air viscosity [8]. Hence, there is an efficiency $\eta$ which is the coupling coefficient between the mechanical input power and acoustic streaming power in the air-mass flown out radially. The output air-mass power is then the product of the static pressure $p$ and volume flow-rate near the fan edge and is related to the input power as -

$$
P_{A}=p D W v_{\amalg}=\eta P_{M}
$$

The air-mass and fan-edge velocities can be estimated and related to each other using the Bernoulli energy equation while including the static pressure energy term produced by the fan. The velocities are related as $v_{\amalg}=\sqrt{2 \eta}\left|v_{\perp}\right|$ for the case of incompressible flow assuming negligible resistance to air flow in the device channel. For an experimentally determined resonance frequency $\omega$, and fan-edge amplitude $y_{0}, v_{\perp}=\omega y_{0}$. Thus, the measured air-velocity directly gives a lower-bound estimate of the power-transfer efficiency $\eta$ from the fan to the air-mass.

In order to compute the air-mass velocity, $f_{\text {air }}$ derived above is substituted in the non-linear equation of motion. The resonance frequency is $\omega=\sqrt{K / M}$ while the amplitude term is solved for using Fourier analysis as in [9]. The forcing function E relates the applied voltage $V$ across the piezoelectric to the fan-edge displacement at resonance which yields the expression for airvelocity [9] as -

$$
v_{\amalg}=C \sqrt{\eta}\left(\frac{d_{31} V}{t_{P Z T} \sqrt{\eta}}\right)^{1 / 3} \frac{y_{0}^{1 / 2}}{L}
$$

Here, $\mathrm{C}$ is constant with appropriate dimensions for lumping together numerical coefficients from the Fourier analysis and the densities of the air and beam materials. Finally the total volume flow-rate can be estimated as -

$$
Q=2 y_{0} W v_{\amalg}
$$

Thus, for thin-film $\mathrm{d}_{31}$ coefficient values of the order of those for

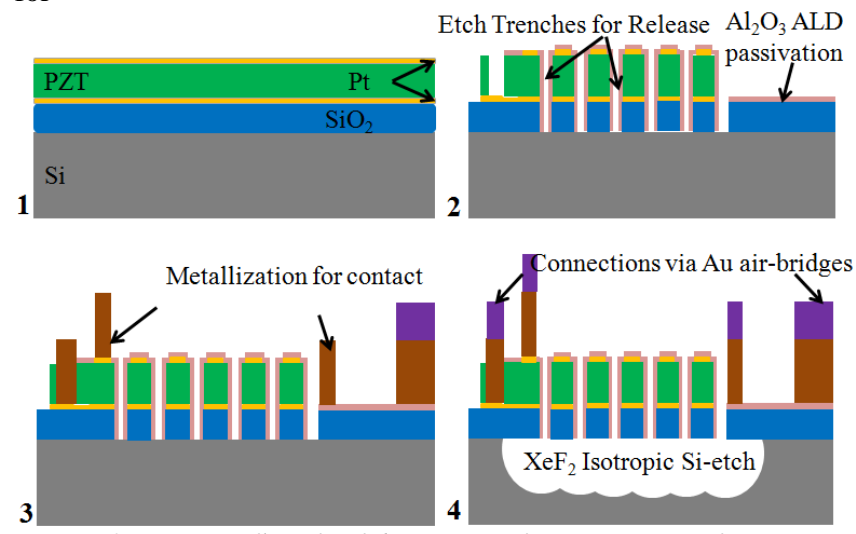

Figure 2: Process flow for fabrication of sense-actuate fan pairs

bulk PZT, the 500nm thickness thin-film devices offer $\sim 30 \mathrm{x}$ enhancement in E-field for the same applied voltages, compared to $0.5 \mathrm{~mm}$ thick bulk PZT, enabling low-voltage operation for comparable flow-rate production.

\section{FABRICATION}

The devices were fabricated using the thin-film PZT growth process at ARL[10] shown briefly in Figure 2. A combination of thermal oxidation, CVD, sputtering, and ion-milling were used to deposit and pattern layers while the PZT films were made via solgel solution deposition, and adding between 0 and $30 \%$ excess lead to account for losses during crystallization. Contact to the top $\mathrm{Pt}$ electrode was made with $\mathrm{Cr} / \mathrm{Pt} / \mathrm{Au}$ air-bridges followed by release via etch-trenches using $\mathrm{XeF}_{2}$ for isotropic $\mathrm{Si}$ etch.

Fabricated devices have a $\mathrm{SiO}_{2}$ elastic layer of $500 \mathrm{~nm}$, bottom electrode of $\mathrm{TiO}_{2} / \mathrm{Pt}$ with thickness $36 \mathrm{~nm} / 100 \mathrm{~nm}, \mathrm{PZT}$ of $500 \mathrm{~nm}$ and top electrode of $100 \mathrm{~nm}$ with gold air-bridges for contact. Sense-actuate fan pairs of widths 200 um each and lengths 800 um and 1000 um with separations of 300 um show in Figure 3 were tested for this report.

\section{PZT CHARACTERIZATION}

The fan edges bend and curve out of the wafer-plane due to residual film stresses after release, a consequence of the long axial dimension. As a result, optical profilometry or laser Doppler vibrometry was not used for direct displacement measurements

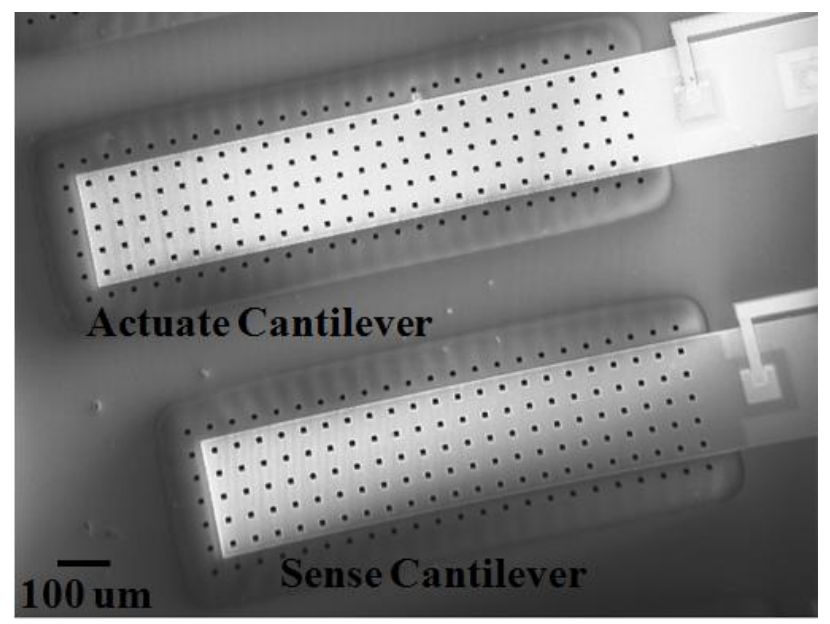

Figure 3: Scanning electron micrograph (SEM) of the unreleased sense-actuate fan-pair 
since the laser light used in these systems does not return back to the detector due to angled incidence on the fan surface, the angle for each fan being a parameter which is difficult to control or tune. Hence for PZT characterization, fans were mounted at 90 degrees to the wafer-plane such that the fan bending, along its thickness when actuated, was in the plane of imaging to optically measure displacements accurately. This was used to measure the $d_{31}$ coefficient of PZT. In this analysis, it was assumed that shear-effects during bending are negligible and residual stress-induced curvature is ignored. DC actuation up to $6 \mathrm{~V}$ was used to measure fan-edge displacement up to $121 \mathrm{um}$ (for $800 \mathrm{um}$ long fan) and $148 \mathrm{um}$ (for 1000 um long fan) following which, multilayer analysis for arbitrary piezoelectric-elastic layer stacks was used to calculate $d_{31}$ [11]. Typical poling field strengths for PZT are of the order of 300 $\mathrm{kV} / \mathrm{cm}[12]$, hence, the actuation voltages are not expected to affect PZT performance or depolarize it. For isotropic Poisson's ratio $v_{i}$, the effective Young's moduli $Y_{i}$ and coupling coefficients $\mathrm{d}_{31, i}$ of the $i$-th layer in the multi-morph stack are modified for this analysis as follows -

$$
\begin{gathered}
Y_{i} \rightarrow Y_{i} /\left(1-v_{i}^{2}\right) \\
d_{31, i} \rightarrow d_{31, i} /\left(1+v_{i}\right)
\end{gathered}
$$

By using the fact that axial forces and moments at any crosssection for a DC-actuated beam are in equilibrium and assuming equal radius of curvature for all layers in the multi-morph, the $d_{31}$ for $\mathrm{PZT}=-85.8 \pm 5 \mathrm{pC} / \mathrm{N}$. This value is less than typical $\mathrm{d}_{31}$ values of bulk PZT-5H close to $-275 \mathrm{pC} / \mathrm{N}$ but within reasonable expectation for multi-layer stressed thin-film devices.

\section{FLOW MEASUREMENTS}

Resonance frequencies for the $800 \mathrm{um}$ and $1000 \mathrm{um}$ fans were measured to be at $614 \mathrm{~Hz}$ and $505 \mathrm{~Hz}$ with $2.5 \mathrm{~mW}$ power input for charging-discharging the PZT capacitance. A commercial resistance temperature detector (RTD) element was used to measure air-velocity as a function of drive frequency. At each drive frequency, the RTD element stabilizes to a peak measured air-velocity which is plotted in Figure 4. The 800 um long fan has produces a peak air-velocity of $7 \mathrm{~cm} / \mathrm{sec}$ at resonance. As expected from the piezo-fan model, this peak air-velocity produced by the fans varies inversely as the fan length. Also, the actuation frequency for peak air-velocity corresponds to the resonance frequency of the fans. Based on the measured fan velocities and fan-edge displacement at resonance, the calculated flow-rates and mechanical to air-mass flow power conversion efficiency are summarized in Table 1.

The piezo-fan model in [9] predicts a flow rate independent of length, assuming the Bernoulli equation holds true and noresistance to air-flow. However, our measurements are done very close to the RTD sensor element which is housed in a metal casing, and is likely to add some resistance for air-flow. Also, the large vibration amplitude 110 um for 800 um fan and 140 um for 1000 um fan, means not all the air-flow is directed radially outwards towards the sensor. Figure 5 plots the voltage dependence of the measured peak air-velocity from the fan. The equation for airvelocity $\mathrm{V}_{\|}$predicts a $\mathrm{V}^{5 / 6}$ voltage dependence - there is a $\mathrm{V}^{1 / 3}$ in the equation derived and a further $V^{1 / 2}$ due to $y_{0}^{1 / 2}$ dependence on edge-displacement since $y_{0}$ is directly proportional to $\mathrm{V}$. The datafit for this dependence are plotted as dotted lines with the expected factor being $1 / \mathrm{L}$ for each curve. Table 2 summarizes the performance of our PZT-based fan and compares it to electrostatically actuated micro-fan and valve-based micro-pumps for chip-scale pumping.

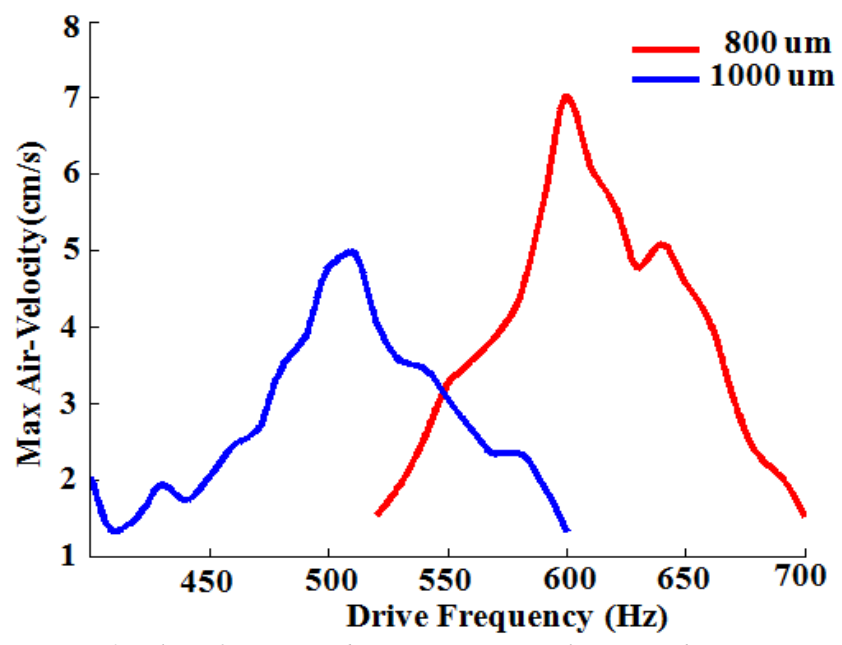

Figure 4: Plot of measured maximum air-velocity with RTD sensor element at the tip of the drive fan as a function of frequency for 800 um and 1000 um long piezofans

Table 1: Summary of flow-velocities, flow rates and mechanical to air-mass flow power transfer efficiencies

\begin{tabular}{|c|c|c|c|}
\hline $\begin{array}{l}\text { Device } \\
\text { Length }\end{array}$ & $\begin{array}{c}\text { Flow } \\
(\mathbf{c m} / \mathbf{s e c})\end{array}$ & $\begin{array}{c}\text { Flow-Rate } \\
(\boldsymbol{\mu L / m i n})\end{array}$ & $\begin{array}{c}\text { Efficiency } \\
(\boldsymbol{\eta})\end{array}$ \\
\hline $800 \mathrm{um}$ & 7.01 & 185.1 & $1.3 \%$ \\
\hline $1000 \mathrm{um}$ & 4.97 & 167 & $0.7 \%$ \\
\hline
\end{tabular}

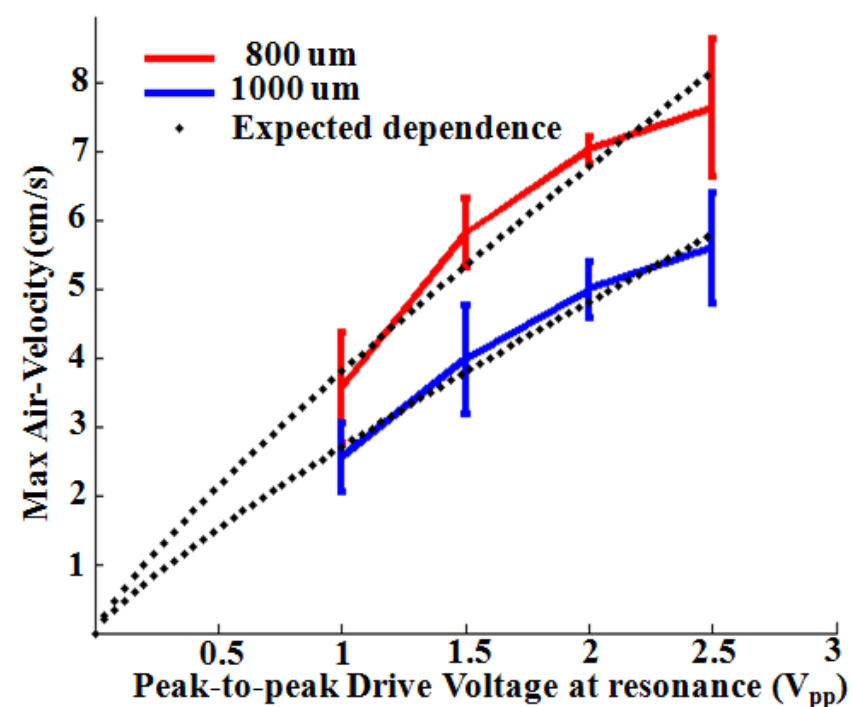

Figure 5: Plot of measured maximum air-velocity with RTD sensor element at the tip of the drive fan and expected $V^{5 / 6}$ dependence

Table 2: Comparison of this work with previous reports for chipscale fans and pumps

\begin{tabular}{|c|c|c|c|}
\hline Work & $\begin{array}{c}\text { Max Flow } \\
(\boldsymbol{\mu L / m i n})\end{array}$ & $\begin{array}{c}\text { Nominal } \\
\text { Voltage }\end{array}$ & $\begin{array}{c}\text { Power } \\
(\mathbf{m W})\end{array}$ \\
\hline $\begin{array}{c}\text { Valve-based } \\
\text { Micropump [5] }\end{array}$ & 4000 & $100 \mathrm{~V}$ & 57 \\
\hline $\begin{array}{c}\text { Electrostatic } \\
\text { Microfan [6] }\end{array}$ & 10 & $100 \mathrm{~V}_{\mathrm{p}-\mathrm{p}}$ & - \\
\hline $\begin{array}{c}\text { Piezo fan - } \\
\text { This work }\end{array}$ & 185.1 & $2 \mathrm{~V}_{\mathrm{p}-\mathrm{p}}$ & 2.5 \\
\hline
\end{tabular}




\section{SENSE FAN MEASUREMENTS}

The novel sensing scheme introduced in our pumping system is the sense fan which measures potential lateral turbulent air-flow, if any, due to large drive on the actuator. To prevent turbulence in micro-channels, inertial fluid forces must be weaker than viscous forces allowing layers of fluid air to slide. Any kind of lateral convection can be considered a signature of turbulence which is where the sense-fan provides a feedback signal via flutter. Our experiments demonstrate a proof-of-concept for this system, rigorous analysis of the turbulent air-flow is not discussed.

In the sense-actuate fan pair testing, the 1000 um long actuate fan was driven with increasing drive amplitude. Correspondingly, the voltage developed across the sense fan due to a force applied by turbulent lateral air-flow, if any, was sensed via the reverse piezoelectric effect. Figure 6 plots direct time-domain signals obtained from the sense-fan flutter for various drive voltages on actuator at the resonance frequency of the actuator. Since the quality factors for the resonant operation of our fans in air are low $(\sim 10)$, precise frequency matching is not required.

Figure 7 plots the DC and AC voltage-signal levels as a function of drive voltage on the actuator at resonance. Clearly, as the drive is increased both a constant force (indicated by the DC level) and a flutter (indicated by the AC level) are seen on the sense and these increase with the drive. The FFT of the signals shows a peak at $1108 \mathrm{~Hz}$, roughly twice the drive frequency and suppressed signal at the drive, perhaps since the force due to lateral air-pressure is modulated at twice the frequency.

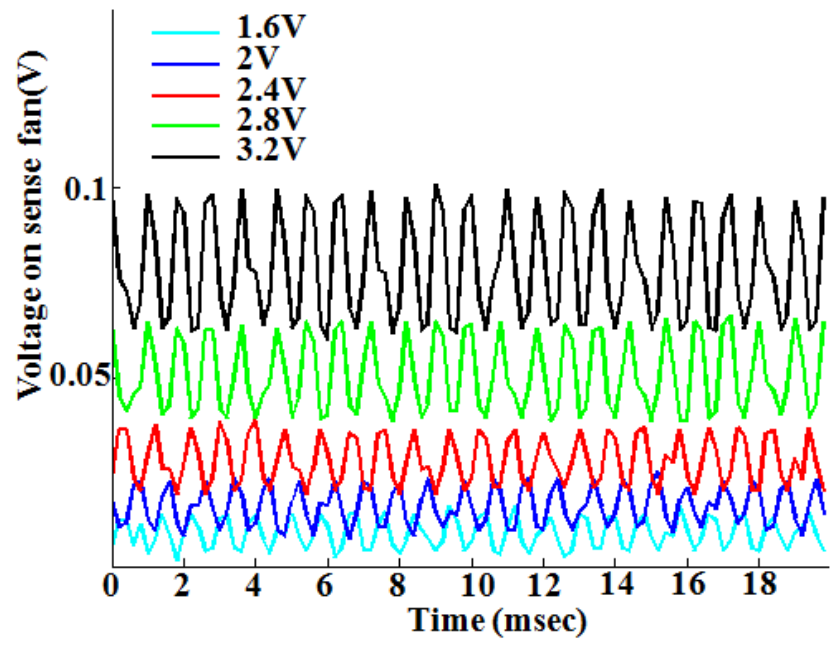

Figure 6: Sense-fan time-domain voltage output for different drive voltages on the actuator at resonance

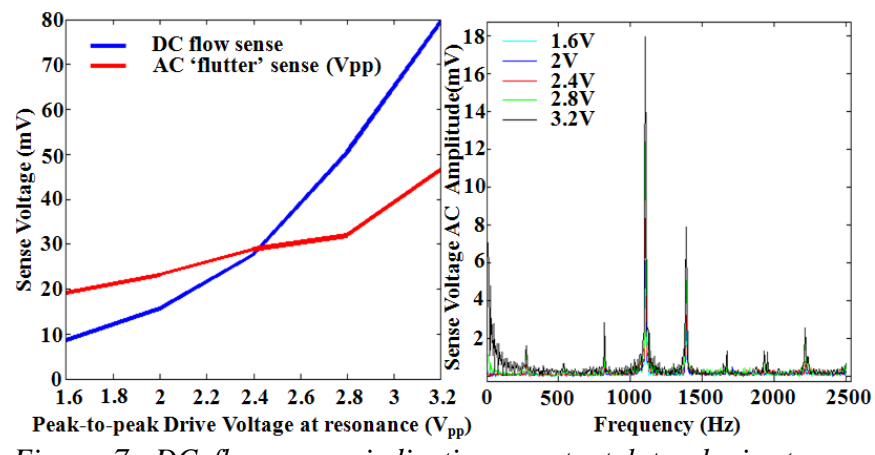

Figure 7: DC flow sense indicating constant lateral air-stream force and AC fluctuation showing increasing flutter/turbulence as actuator drive is increased and FFT of the AC signals with a peak at $1108 \mathrm{~Hz}$, which is twice the resonant drive frequency

\section{CONCLUSIONS}

In this paper, we have presented a novel low-voltage senseactuate piezo-fan pair for potential integration in chip scale gas sensing applications. The thin-film PZT process enables scaling to achieve required electric fields with low voltage operation, for CMOS compatible operation. The sense-fan allows for feedback turbulent flow-sensing without complicated circuitry and extensive additional on-chip real-estate. By integrating the fan with ionmobility-based sensors as in [7] and benign electron-emitting ${ }^{63} \mathrm{Ni}$ for ionization and self-powering as previously demonstrated [13], the system can have self-sustained performance for long periods limited only by the ionizer half-life.

\section{ACKNOWLEDGEMENTS}

The authors would like to acknowledge the ARL fabrication facility for assisting in device fabrication and Cornell Nanofabrication Facility (CNF) for post-processing development and testing equipment. Travel support has been generously provided by the Transducers Research Foundation (TRF).

\section{REFERENCES}

[1] X. Liu et al., "A Survey on Gas Sensing Technology," Sensors, pp. 9635-9665, 2012

[2] R. S. Jayashree et al., "Air-Breathing Laminar Flow-Based Microfluidic Fuel Cell," J. of American Chemical Society, pp. 16758-59, 2005

[3] S. M. Wait et al., "Piezoelectric Fans Using Higher Flexural Modes for Electronics Cooling Applications," IEEE Trans. on Components and Packaging Technologies pp. 119-128, 2007

[4] R. Linderman et al., "Development of the micro rotary fan," Sensors and Actuators A: Physical, Vol. 95 pp. 135-142, 2002

[5] H. Kim et al., "“"A Fully Integrated High-Efficiency Peristaltic 18-Stage Gas Micropump with Active Valves," IEEE MEMS, pp. 131-134, 2007

[6] R. Linderman et al., "The resonant micro fan gas pump for active breathing microchannels," IEEE Transducers, Vol. 2 pp. 1923-1926, 2003

[7] V. Gund et al., "Low-voltage $(<5 \mathrm{~V})$ ion-mobility spectrometer array for label-free gas detection," IEEE Transducers, pp. 2767-2770, 2013

[8] A. Ihara and H. Watanabe , "On the Flow Around Flexible Plates, Oscillating with Large Amplitude," J. of Fluids and Structures, Vol. 8, pp. 601-619, 1994

[9] M. Toda, "Theory of air flow generation by a resonant type PVF2 bimporh cantilever vibrator," Ferroelectrics, Vol. 2 pp. 911-918, 1978

[10] T.G. Ivanov et al., "Analog RF MEMS Attenuator with PZT Actuators," Transducers, pp. 2767-2770, 2013

[11] D. L. DeVoe and A. P. Pisano, "Modeling and Optimal Design of Piezoelectric Cantilever Microactuators," J. of Microelectromechanical Systems, Vol. 6 pp. 266-270, 1997

[12] G. Park et al., "Measurement of piezoelectric coefficients of lead zirconate titanate thin films by strain-monitoring pneumatic loading method," Applied Physics Letters, Vol. 8, pp. 4606-4608, 2002

[13] R. Duggirala et al., "Radioisotope Thin-Film Fueled Microfabricated Reciprocating Electromechanical Power Generator," J. of Microelectromechanical Systems, Vol. 7, pp. 837-849, 2008

\section{CONTACT}

*Ved Gund, tel: +1-650-521-1172; vvg3@ cornell.edu 